\title{
Relapsed Multiple Myeloma Presenting as Intracranial Plasmacytoma and Malignant Pericardial Effusion following Recent Allogeneic Stem Cell Transplantation
}

\author{
Jonathan Pan ${ }^{\mathrm{a}} \quad$ Jason Chen $^{\mathrm{a}} \quad$ Joanne Filicko $^{\mathrm{a}} \quad$ Dolores Grosso $^{\mathrm{a}}$ \\ Michelle Nagurney ${ }^{b} \quad$ Onder Alpdogan $^{a} \quad N^{2}$ al Flomenberg ${ }^{a}$ \\ a Department of Medical Oncology, Thomas Jefferson University Hospital, \\ Philadelphia, PA, USA; ${ }^{b}$ Department of Pathology, Thomas Jefferson University Hospital, \\ Philadelphia, PA, USA
}

\section{Keywords}

Multiple myeloma $\cdot$ Intracranial plasmacytoma $\cdot$ Malignant pericardial effusion $\cdot$ Stem cell transplant

\begin{abstract}
Although rare, both central nervous system and pericardial involvement of myeloma have been well described in the literature. Their simultaneous occurrence in relapsed disease, however, has not been previously reported. This case describes a 54-year-old female who was treated for high-risk multiple myeloma with multiregimen chemotherapy and allogeneic hematopoietic stem cell transplantation. Four months after transplant, she was found to have relapsed disease manifesting as an extraosseous, intracranial plasmacytoma and simultaneous malignant pericardial effusion. Her disease characteristics, treatment course, radiologic and pathologic findings are described in detail, and we review the previous literature to determine the various aspects of her disease that may have contributed to her aggressive clinical course.


 Oncology}

\section{Introduction}

Central nervous system (CNS) involvement of multiple myeloma is rare, with an incidence rate reported around 1\% [1, 2]. Most of these cases involve lepto-meningeal disease, with intracranial plasmacytoma being less frequent, especially following treatment of myeloma [3]. Similarly, malignant pericardial effusion from extramedullary multiple myeloma is uncommon, especially following hematopoietic stem cell transplantation (HSCT) [4]. We present a unique case of relapsed multiple myeloma manifesting as an extraosseous, solitary intracranial plasmacytoma and simultaneous malignant pericardial effusion within 4 months of allogeneic HSCT.

\section{Case Presentation}

Our patient is a 54-year-old female with a past medical history of IgG kappa multiple myeloma diagnosed after workup of bone pain affecting her shoulders and ribs. At the time of diagnosis, her serum protein electrophoresis (SPEP) showed an M-spike with monoclonal IgG kappa concentration of $3.5 \mathrm{gm} / \mathrm{dL}$. Total serum IgG was elevated at 5,805 mg/dL, $\beta_{2} \mathrm{mi}-$ croglobulin was $2.3 \mathrm{mg} / \mathrm{L}$, albumin was $3.9 \mathrm{~g} / \mathrm{dL}$, and LDH was elevated at $272 \mathrm{IU} / \mathrm{L}$. She was diagnosed with International Staging System stage I disease. Cytogenetic analysis revealed translocation (4;14), trisomy 9, and monosomy 13 with a complex karyotype, suggesting high-risk disease.

She received 1 cycle of lenalidomide and dexamethasone followed by radiation therapy including 30 gray in 10 fractions to the jaw, spine, and right cavernous sinus. She then received 4 cycles of lenalidomide, bortezomib, and dexamethasone but was noted to have increased bone disease in her ribs, femur, and hip, so she received 3 cycles of carfilzomib, lenalidomide. and dexamethasone along with 30 gray in 10 fractions to her femur and ribs, and 16 gray in 4 fractions to both hips. Follow-up $\kappa$ - and $\lambda$-light chain values and IgG level were normal, and SPEP showed a small residual M-spike which was too small to quantify.

Her bone marrow biopsy showed $<1 \%$ clonal plasma cells. Due to her age and high-risk disease, she was offered a reduced-intensity allogeneic HSCT. She underwent a two-step haplo-identical HSCT approximately 1 year after initial diagnosis, utilizing a fludarabine, busulfan, and cyclophosphamide conditioning regimen. During that admission, she had a non-contrast head CT that did not show any intracranial pathology.

Two months after HSCT, she was found to have plasmacytoma of the thoracic spine and underwent additional radiation therapy with good response. Bone marrow biopsy at that time showed complete donor engraftment and was negative for abnormal cells. Her SPEP showed a low monoclonal IgG kappa level of $0.2 \mathrm{gm} / \mathrm{dL}$.

Three months following HSCT, she developed intractable nausea, vomiting, and a thirtypound weight loss. A gastrointestinal work up, including barium swallow study, endoscopy, and colonoscopy, was unremarkable and did not suggest infectious etiology or graft-versushost disease. Due to worsening symptoms, she was readmitted for further evaluation. On admission, she denied any focal neurologic deficits or visual complaints, although she reported thoracic paresthesias. Spinal MRI revealed new thoracic soft tissue lesions, and a brain MRI revealed a new extra-axial right occipital lobe mass extending to the cerebellum (Fig. 1).

The patient underwent right craniotomy with partial resection of the brain lesion. Grossly, the tumor was extraosseous with a distinct plane in the occipital pole causing dis- 


\section{Case Reports in Oncology}

placement of the tentorium but without obvious infratentorial spread. Pathology revealed CD138-positive cells consistent with plasmablastic plasmacytoma (Fig. 2). She underwent cranial and spinal irradiation with improvement of her pain, paresthesias, and intractable nausea.

During the same admission, she was found to have a large pericardial effusion which was incidentally seen on a CT thorax. She denied cardiac or respiratory complaints and was hemodynamically stable without pulsus paradoxus. The effusion was monitored with serial echocardiograms, eventually enlarging in size and developing tamponade physiology. This led to immediate placement of a pericardial drain, resulting in $1 \mathrm{~L}$ of bloody drainage. Fluid analysis revealed CD138-positive plasma cells. Due to the rapid progression of her disease and multi-organ involvement after prior multi-agent chemotherapy and HSCT, the patient and her family decided on hospice care.

\section{Discussion}

This case demonstrates a rare presentation of very rapidly relapsing multiple myeloma in the form of extraosseous, solitary plasmacytoma and malignant pericardial effusion, which has not been previously reported in the literature. In a recent retrospective review of 172 CNS myeloma patients by Jurczyszyn et al. [5], the median time from multiple myeloma diagnosis to CNS involvement was 25 months. Our patient was found to have an intracranial plasmacytoma 4 months after allogeneic HSCT. Though less sensitive than MRI, her head CT at the time of transplant did not detect an intracranial abnormality. Her prior bone marrow biopsy after HSCT was negative for disease, and her most recent SPEP showed a low level of monoclonal protein. Despite the size and location of the lesion, the patient did not present with typical CNS manifestations, such as altered mental status, gait abnormality, vision changes, or focal neurologic deficits. Her only symptomatic manifestations were pain, paresthesias affecting her thoracic region (likely reflecting her spine disease), intractable nausea, and vomiting. These symptoms improved after tumor resection and radiation therapy.

There is limited data on intracranial plasmacytoma in patients following allogeneic HSCT. In patients who have received autologous HSCT, most cases of CNS relapse are leptomeningeal disease $[6,7]$. There were no cases of solitary intracranial plasmacytoma in myeloma patients after autologous HSCT. Overall, CNS involvement of multiple myeloma was previously reported in around $1 \%$ of all multiple myeloma cases [1], and it was found to be $0.7 \%$ in a recent Mayo Clinic study of 4,060 myeloma patients over 16 years [2]. The majority of CNS cases are found in relapsed disease; however, most of these cases typically present as a leptomeningeal rather than an intraparenchymal process. Several cases of solitary intracranial plasmacytoma have been reported, but these are typically intraosseous tumors [3, 8-10]. Extraosseous intracranial plasmacytoma is rarely seen with relapsed disease. Our patient's tumor displayed similar MRI findings as reported in other known intraosseous cases, mainly homogenous enhancement with perilesional edema, with features mimicking lymphoma or meningioma [11].

Prior studies on CNS and extramedullary myeloma have identified alterations of chromosome 13 and 17, and translocation $(4 ; 14)$ as higher risk features $[1,12]$. Elevated LDH was also seen more frequently in these patients along with IgA and IgD subtypes of myeloma. Our patient showed similar characteristics of chromosome 13 deletion, translocation $(4 ; 14)$, and an elevated LDH. Her myeloma type, however, was IgG kappa light chain. Her plasmacytoma displayed CD138-positive marker, seen in most CNS myeloma cases, and had 
a high percentage of Ki-67 positivity which is associated with extraosseous relapse of myeloma [12].

The prognosis of CNS myeloma is poor, with the average overall survival of 3-6 months $[2,5]$. There is no standard of care, although a recent study by Jurcyzszyn et al. [5] concluded that systemic chemotherapy with or without radiation therapy improved survival duration over patients who were treated without systemic chemotherapy.

Malignant pericardial effusion from extramedullary multiple myeloma is a rare finding occurring in $<1 \%$ of all myeloma cases [4]. There has been a small case series and case reports of myeloma relapse presenting as malignant pericardial effusion $[4,13]$; however, only a single case was after allogeneic HSCT [14]. Malignant effusion from myeloma carries a particularly poor prognosis as the average survival duration was calculated at 13.5 weeks after diagnosis. In this series, treatment with chemotherapy led to a high rate of death from sepsis. Aside from drainage of symptomatic effusions, there is no optimal management guideline.

Due to the rapid progression of disease and both neurologic and cardiac involvement, our patient declined systemic chemotherapy. She received palliative brain and spinal radiation, and ultimately opted to pursue hospice care. She died 1 month later.

Even with the knowledge of her high-risk cytogenetics, this case of myeloma relapse was profoundly rapid and aggressive. Following HSCT, she demonstrated a small disease burden on routine laboratory surveillance. The fact that she relapsed with simultaneous intracranial plasmacytoma and malignant pericardial effusion is unusual, likely reflecting an aggressive myeloma which had already failed treatment with multiple chemotherapy agents. Given the substantial growth in the number of effective treatments available for multiple myeloma, patients failing a larger number of agents may demonstrate more aggressive and unusual patterns of recurrent disease.

\section{Statement of Ethics}

The authors have no ethical conflicts to disclose.

\section{Disclosure Statement}

The authors have no conflicts of interest to disclose.

\section{References}

1 Fassas AB, Muwalla F, Berryman T, et al: Myeloma of the central nervous system: association with highrisk chromosomal abnormalities, plasmablastic morphology and extramedullary manifestations. $\mathrm{Br} \mathrm{J}$ Haematol 2002;117:103-108.

2 Paludo J, Painuly U, Kumar S, et al: Myelomatous involvement of the central nervous system. Clin Lymphoma Myeloma Leuk 2016;16:644-654.

- 3 Wilberger AC, Prayson RA: Intracranial involvement by plasma cell neoplasms. Am J Clin Pathol 2016;146:156-162.

-4 Abelman W, Virchis A, Yong K: Extramedullary myeloma representing as a pericardial effusion with tamponade: two case reports and a further review of 19 cases in the literature. Leuk Lymphoma 2005;46:137-142.

-5 Jurczyszyn A, Grzasko N, Gozzetti A, et al: Central nervous system involvement by multiple myeloma: a multi-institutional retrospective study of 172 patients in daily clinical practice. Am J Hematol 2016;91:575-580. 


\section{Case Reports in Oncology}

\begin{tabular}{l|l}
\hline Case Rep Oncol 2017;10:582-587 \\
\hline DOI: 10.1159/000478001 & $\begin{array}{l}\text { C } 2017 \text { The Author(s). Published by S. Karger AG, Basel } \\
\text { www.karger.com/cro }\end{array}$ \\
\hline
\end{tabular}

Pan et al.: Relapsed Multiple Myeloma Presenting as Intracranial Plasmacytoma and Malignant Pericardial Effusion following Recent Allogeneic Stem Cell Transplantation

-6 Petersen SL, Wagner A, Gimsing P: Cerebral and meningeal multiple myeloma after autologous stem cell transplantation. A case report and review of the literature. Am J Hematol 1999;62:228-233.

7 Veinstein A, Brizard A, Randriamalala E, Babin P, Preud'homme JL, Guilhot F: Central nervous system relapses after autologous stem cell transplantation for myeloma. Report of two cases. Hematol Cell Ther 1997;39:327-330.

-8 Waterhouse DF, Moloney GA, Gargoum FS, Hayden PS, O'Gorman T: Extramedullary relapse of IgAlambda myeloma after recent bortezomib therapy: a case report. Cases J 2009;2:7456.

-9 Daghighi MH, Poureisa M, Shimia M, Mazaheri-Khamene R, Daghighi S: Extramedullary plasmacytoma presenting as a solitary mass in the intracranial posterior fossa. Iran J Radiol 2012;9:223-226.

-10 Zhang MJ, Su GH, Shen J, et al: Multiple myeloma with intracranial extension and bilateral renal infiltration: a case report and review of the literature. Oncol Lett 2015;10:2337-2340.

11 Patriarca F, Zaja F, Silvestri F, et al: Meningeal and cerebral involvement in multiple myeloma patients. Ann Hematol 2001;80:758-762.

12 Rasche L, Bernard C, Topp MS, et al: Features of extramedullary myeloma relapse: high proliferation, minimal marrow involvement, adverse cytogenetics: a retrospective single-center study of 24 cases. Ann Hematol 2012;91:1031-1037.

13 Arat M, Ulusoy V, Demirer T, et al: An unusual presentation of plasma cell dyscrasias: cardiac tamponade due to myelomatous infiltration. Leuk Lymphoma 2002;43:145-148.

$\$ 14$ Zeiser R, Hackanson B, Bley TA, Finke J, Bertz H: Unusual cases in multiple myeloma and a dramatic response in metastatic lung cancer: Case 1. Multiple myeloma relapse presenting as malignant pericardial effusion. J Clin Oncol 2005;23:230-231.

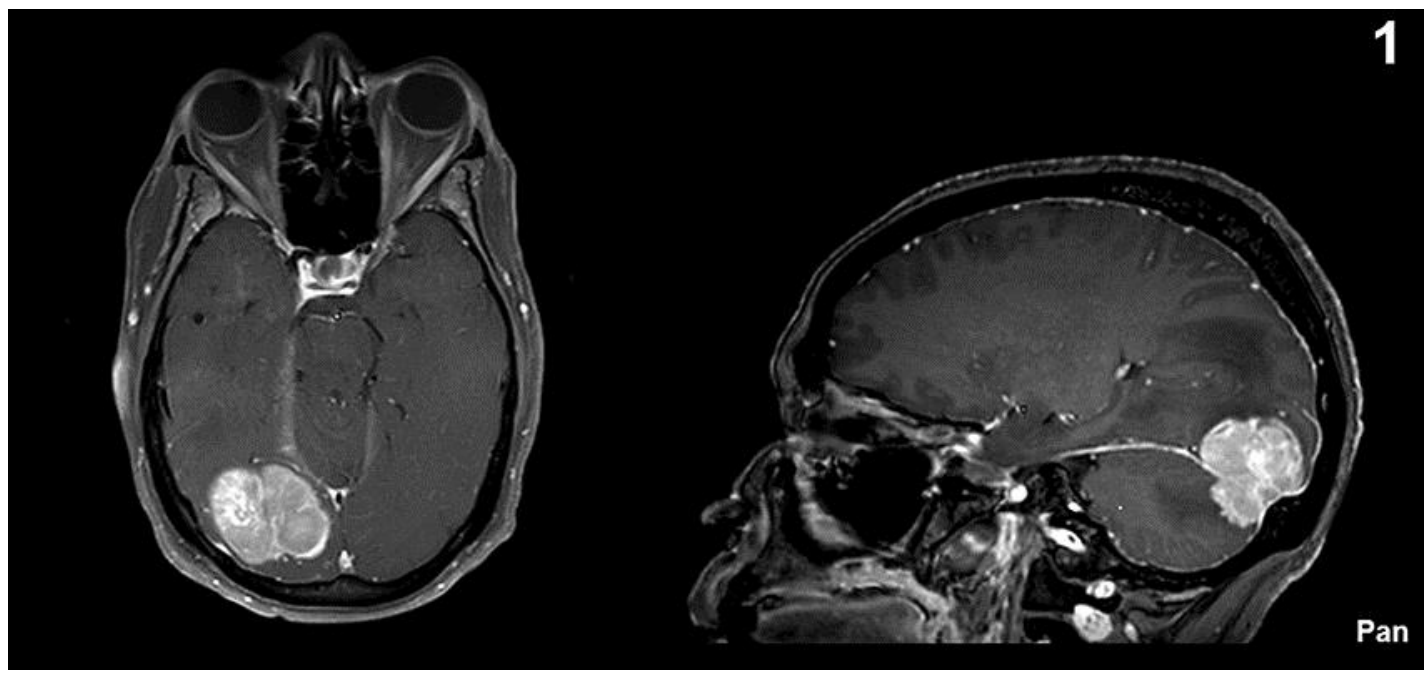

Fig. 1. A $3.5 \times 4.2 \times 2.6 \mathrm{~cm}$, homogeneously enhancing, extra-axial mass along the posterior right occipital lobe and right cerebellum with associated parenchymal edema. The differential diagnosis based on history and radiographic appearance includes plasmacytoma, metastatic disease, lymphoma, or aggressive meningioma. 


\section{Case Reports in Oncology}

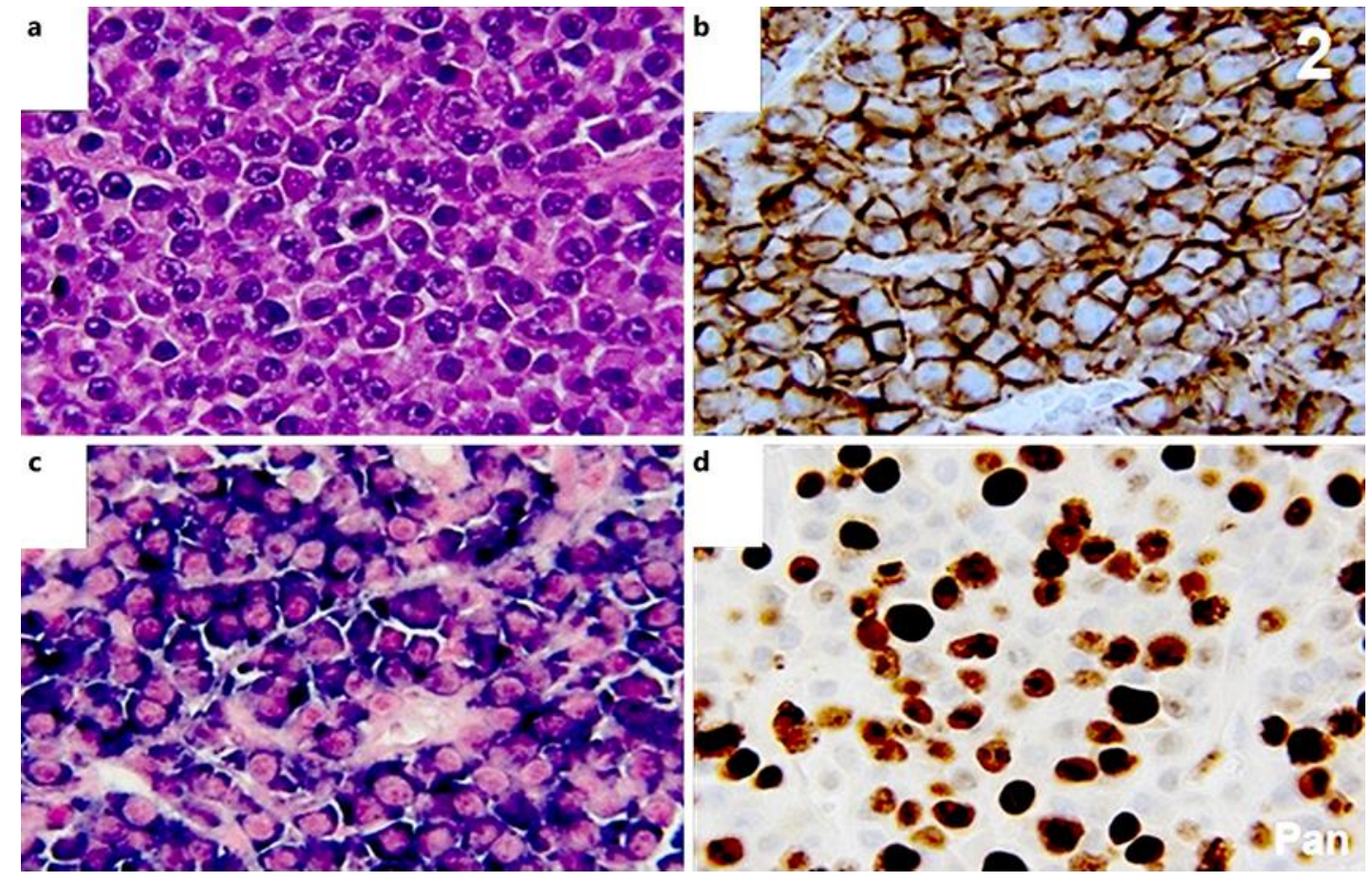

Fig. 2. Brain biopsy. a Hematoxylin and eosin staining reveals sheets of neoplastic plasma cells with large nuclei and prominent nucleoli, and mitotic figures. b By immunohistochemistry (IHC), the cells strongly express CD138, a specific marker for plasma cells. c In situ hybridization stain additionally shows kappa (к) light chain restriction. $\mathbf{d}$ The cells are robustly proliferating, expressing Ki-67, a specific marker for cell proliferation, in $56 \%$ of the neoplastic cells. 